\title{
Diagrammatic Representation as a Tool for Clarifying Logical Arguments
}

\author{
Sabah Al-Fedaghi \\ Computer Engineering Department \\ Kuwait University \\ Kuwait
}

\begin{abstract}
Knowledge representation of reasoning processes is a central notion in the field of artificial intelligence, especially for knowledge-based agents, because such representation facilitates knowledge of action outcomes necessary for optimum performance by problem-solving agents in complex situations. Logic is the primary vehicle by which knowledge is represented in knowledge-based agents. It involves logical inference that produces answers from what is known based on this inference mechanism. Modus Ponens is the best-known rule of inference that is sound. Recently, a dispute has arisen regarding attempts to show that modus ponens is not a valid form of inference. Part of the cause of the controversy is miscommunication of the involved problem. This paper proposes a diagrammatic representation of modus ponens with the hope that such a representation will serve to clarify the issue. The advantage of this diagrammatic representation is a better understanding of the reasoning process behind this inference rule.
\end{abstract}

Keywords - artificial intelligence; diagrammatic representation; conditionals; argument forms; logical argumentation; modus ponens

\section{INTRODUCTION}

This paper is concerned with the representation of knowledge and the reasoning process, which are central notions in artificial intelligence, especially for knowledgebased agents. The subject is important for artificial agents because they facilitate knowledge of action outcomes necessary for their optimum performance in complex situations and partially observable environments.

Logic is a primary vehicle for representing knowledge. It involves logical inference that produces answers from what is known based on this inference mechanism. In addition to this function of reasoning, an inference mechanism can be utilized for self-learning by artificial agents. Knowledge bases founded on logic consist of statements that accept truth-values with respect to each possible world. They also involve logical entailment between statements, where statements follow logically from other statements. Entailment can be used to derive conclusions - that is, to carry out logical inference. An inference rule that derives only entailed sentences is said to be sound or truth preserving.

Modus Ponens (MP) is the best-known rule of inference that is sound or truth preserving and hence can be applied to derive conclusions that lead to the desired goal. History-wise, it has been considered one of the five basic inference rules that are valid without proof (e.g., by the Stoics [1]). Currently, it is still a central tool; for example, MP is an important forwardchaining inference in a knowledge base of Horn clauses to determine whether a statement is entailed by the knowledge base.

This paper focuses on such MP because recent attempts have been made to show that modus ponens is not a valid form of inference. The paper does not counter or support the logical argument of such an attempt. The underlying thesis is that representation has contributed to such a controversy. This paper aims at proposing a diagrammatic representation of modus ponens with the hope of clarifying the issue in relation to MP.

Current methods of diagrammatic representation of logic formulas incompletely depict the underlying semantics of the formulas, creating a conceptual gap that sometimes causes misinterpretation. The methodology proposed in this paper applies a schematizing of logic formulas in the context of modus ponens. The advantage of this diagrammatic representation is better understanding of the reasoning process at the base of this inference rule.

\section{BACKGROUND OF MODUS PONENS}

In logic, an indicative conditional statement is a statement that describes implications or hypothetical situations and their consequence, such as If $p$ then $q$, where $p$ is called the antecedent, and $q$ the consequent; however, in general, such as in natural language, conditional statements are not restricted to this format. In the context of logic, and based on truth conditions, If $p$ then $q$, denoted as $\mathrm{p} \rightarrow \mathrm{q}$, is false when $\mathrm{p}$ is true and $q$ is false, otherwise, it is true. "Conditional sentences have attracted concentrated attention of philosophers, although intermittent, since ancient times..." [2].

On the other hand, MP as a principle of inference expresses that: from the conditional if $p$ then $q$ together with its antecedent $\mathrm{p}$, it can be inferred that q. MP is commonly recognized as a basic rule of inference. Along with MP is the Modus Tollens (MT) rule: "we teach them [MP and MT] in introductory logic courses, related to conditional statements. In everyday reasoning, MP and MT can also have important roles, in modes of argumentation" [2]. As mentioned, attempts have been made to show that MP is not a valid form of inference, and these have been based mainly on a number of counterexamples, thus challenging the accepted view in logic that inferences grounded on MP are deductively valid [3-4]. McGee [5] presents the following MP counterexamples: 
Opinion polls taken just before the 1980 election showed the Republican Ronald Reagan decisively ahead of the Democrat Jimmy Carter, with the other Republican in the race, John Anderson, a distant third. Those apprised of the poll results believed, with good reason:

a) If a Republican will win the election, then if Reagan will not win, Anderson will win.

\section{b) A Republican will win the election.}

c) So, if Reagan will not win, Anderson will win.

But, those apprised of the poll results "did not have reason to believe" conclusion c [5]. This means that $\mathrm{c}$ is not obviously true. This application of MP to an ordinary statement leads to a conclusion that is contrary to common-sense expectation. Accordingly, in light of examples such as this, modus ponens is not strictly valid; see discussions in [6-8]. "McGee's [5] attempt to show that modus ponens is not a valid form of inference - and to show this by the help of a counterexample and not by envisaging an evil demon confusing us - is proof of the ingenuity of a philosopher's ability to doubt" [9]. MacFarlane [10] gives two additional examples, as follows:

(a) If that creature is a fish, then if it has lungs, it is a lungfish. (b) That creature is a fish. (c) So, if it has lungs, it is a lungfish.

(a) If Uncle Otto doesn't find gold, then if he strikes it rich, he will strike it rich by finding silver. (b) Uncle Otto won't find gold. (c) So, if Uncle Otto strikes it rich, he will strike it rich by finding silver.

This paper demonstrates diagrammatic construction of MP for the purpose of producing a conceptually complete description of the involved phenomena. The description can provide illustrations and models that might help in facilitating understanding of the MP-based reasoning process. The approach utilizes a diagrammatic apparatus called the Flowthing Model that, for the sake of completeness, will be briefly described in the next section [11-15].

\section{USING THE FLOWTHING MODEL}

The Flowthing Model (FM) can be related to the notion of fluidity within a web of interrelated flows that cross boundaries of intersecting and nested spheres. This representation is an apparatus that facilitates flowages (acts of flowing). Ingredients in a flowage include flowthings (things that flow), and flow systems (flowsystems). So-called objects, concepts, entities, and time are flowthings. A "thing" is defined as a flowthing: "what is created, released, transferred, arrives, is accepted, and processed" while flowing within and among spheres. In spite of use of the term "thing," the fundamental ontology in FM is that "systems are not composed of things, but are rather defined on things, and there is a clear distinction between their physical 'thinghood' and logical 'systemhood' properties" [16]. Accordingly, a sphere or subsphere can be any object, any region of logical space that is set apart (mentally) from anything else [16].

A flowthing has a permanent identity but impermanent form. A flowsystem constrains the trajectory of flow of flowthings. A particular flowsystem provides the space/time for happenings and existence of flowthings. To flowthings, the flowsystem is formed of six discontinuities: being created, being released, being transferred, being arrived, being accepted, and being processed.

Flows connect six stages that are exclusive for flowthings; i.e., a flowthing can be in one and only one of these six states at a time: Transfer, Process, Creation, Release, Arrival, and Acceptance, as shown in Fig. 1. Where appropriate, we can use Receive as a combined stage of Arrive and Accept. These stages are the elementary basic actions. A system manifests itself by engaging in these actions: processing, creating, releasing, receiving, and transferring of flowthings. In Fig. 1, we assume irreversibility of flow, e.g., released flowthings flow only to Transfer.

Note that this conceptualization of stages as elementary actions may not coincide with other uses of such terms, e.g., in physics. For example, (model) time and (model) space are simply flowthings in FM that can be created, processed, released, etc.; e.g., a clock is a flowsystem that can create, release, and transfer time.

The lower-level spheres where the flows occur are called flowsystems; these include, at most, six stages, as follows:

- Arrive: a flowthing reaches a new flowsystem

- Accepted: a flowthing is permitted to enter the system.

- Processed (changed in form): the flowthing passes through some kind of transformation that changes its form but not its identity (e.g., compressed, colored, compared)

- Released: a flowthing is marked as ready to be transferred (e.g., airline passengers waiting to board after completing processing)

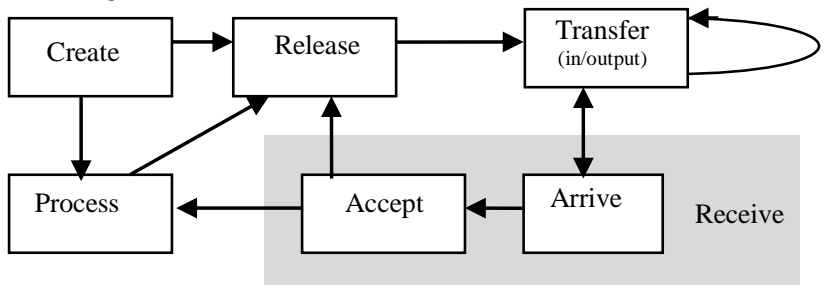

Fig. 1. Flow system

- Created: a new flowthing emerges (comes into existence relative to its sphere) in the system (e.g., processing of a neutron generates a proton, electron, and neutrino)

- Transferred: the flowthing is en route to somewhere outside the flowsystem (e.g., packets reaching ports in a router, but still not in the arrival buffer).

An additional stage of Storage can also be added to any FM model to represent the storage of flowthings; however, storage is a generic stage, not specific, because there can be stored processed flowthings, stored created flowthings, and so on.

A flowsystem may not need to include all the stages because the other stages are irrelevant, have no impact, or are prohibited, e.g., an archiving (storage) system might use only the stages arrive, accept, release, and transfer. Multiple systems captured by FM can interact with each other by triggering interrelated events in their spheres and stages. 


\section{DESCRIBING FORMULAS IN FM}

In FM, a formula $\mathrm{p}$ can be conceptualized as a sphere formed from two subsystems (Body, Truth), as shown in Fig. 2. Consider the statement A Republican will win the election as declared in a logical argument:

1) $\quad \ldots$

2) $\quad \ldots$

3) $p$

This indicates that the inference rule, say, MP, processed the premises and reached the conclusion that triggered the creation of $p$ (circles $a$ and $b$ in Fig. 3). As a result of this creation "(3) p" appears in the chain of deduction with its two flowsystems of truth-value and body (c and d).

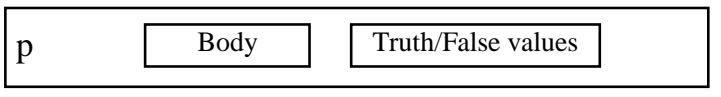

Fig. 2. p as a sphere with two flowsystems

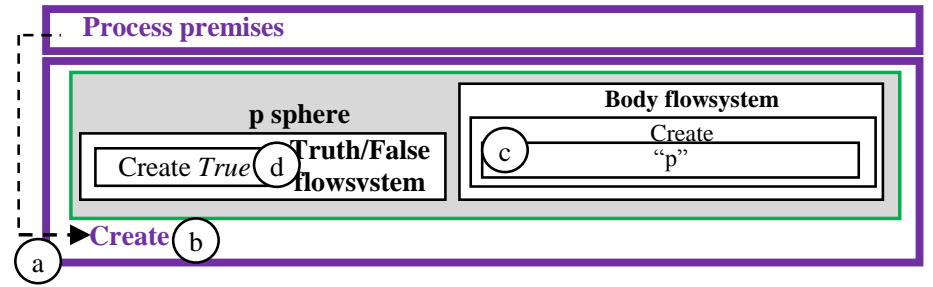

Fig. 3. $\mathrm{p}$ is created as a result of processing premises

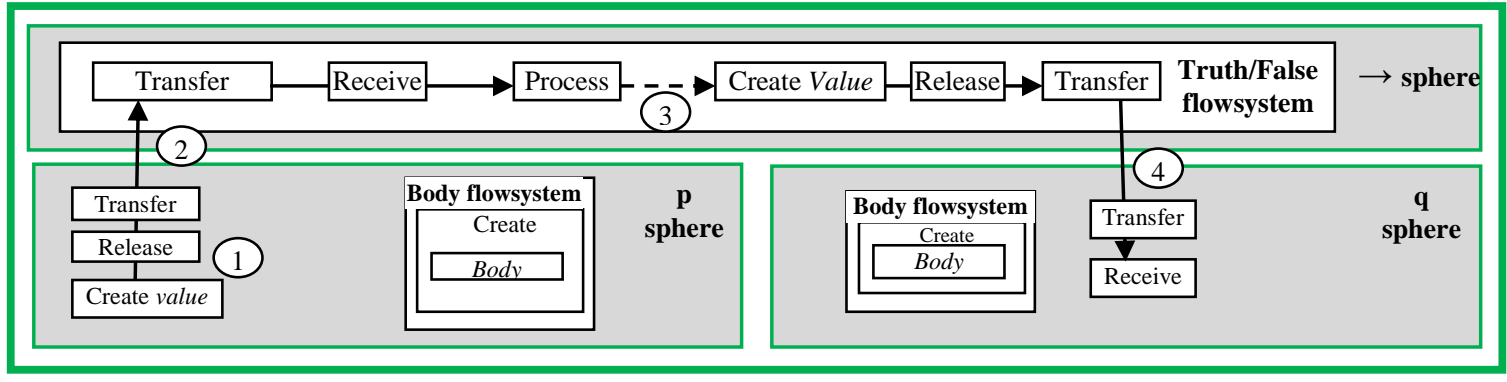

Fig. 4. FM representation of the implication $\boldsymbol{p} \rightarrow \boldsymbol{q}$

Fig. 4 shows the FM representation of the implication $\mathrm{p} \rightarrow \mathrm{q}$ which is formed from p', q', and $\rightarrow$. For simplicity's sake, the truth-value flowsystem (circle 1 in the figure) is not enclosed in a box. The truth-value flows to $\mathrm{p}^{\prime}$, then to the implication (2), where it is processed and, according to the material implication truth table, triggers (3) the creation of a truth-value. This truthvalue flows to q' (4). Note that Fig. 4 is an "empty shell" of structure that will be filled when it is triggered. The implication includes $\mathrm{p}^{\prime}$ and $\mathrm{q}^{\prime}$ as shells (place holders) of structure (no assigned truth-values).

Now consider that the modus ponens:

4) $p \rightarrow q$

5) $p$

is applied to produce $q$ as shown in Fig. 5. Note that the sphere of the MP involves $\mathrm{p}, \mathrm{p} \rightarrow \mathrm{q}$, and $\mathrm{q}$. Such a structure of MP (see Fig. 5) is activated (created) and processed. The antecedent and consequent p' and q' in the figure are "shells" or "place holders" for p and q (loaded with truth-values), in the same way one could give a value to variable $x$ in $x+200$. When $\mathrm{p}$ is created then, if its body is similar to the $\mathrm{p}^{\prime}$, the truthvalue flows and reaches $\rightarrow$ to trigger filling of q'; accordingly, the MP "gives birth to" (creates) q.
Fig. 6 shows the complete FM representation of MP, which involves the following:

a) The creation of $p \rightarrow q$ (shells $p^{\prime}$ and $q^{\prime}$, and $\rightarrow$ ), $p$ and $q(1,2$, and 3, respectively). Note that true is assigned to the $\rightarrow$ sphere (2), activating it, analogous to switching an engine ON, as shown in Fig. 7.

b) Assigning a truth-value to $p$ (1)

Accordingly, the body of $\mathrm{p}$ flows to its corresponding body of the antecedent in the implication (5). If the two bodies are identical (6), then this triggers (7) the flow of truth-value (8 and 9) to the implication to be processed (10) according to the implication truth table.

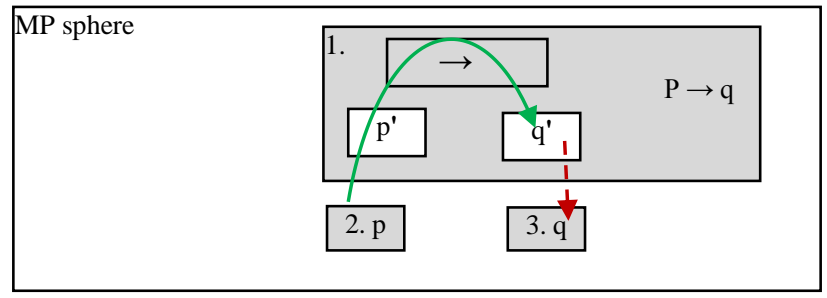

Fig. 5. The sphere of the MP 


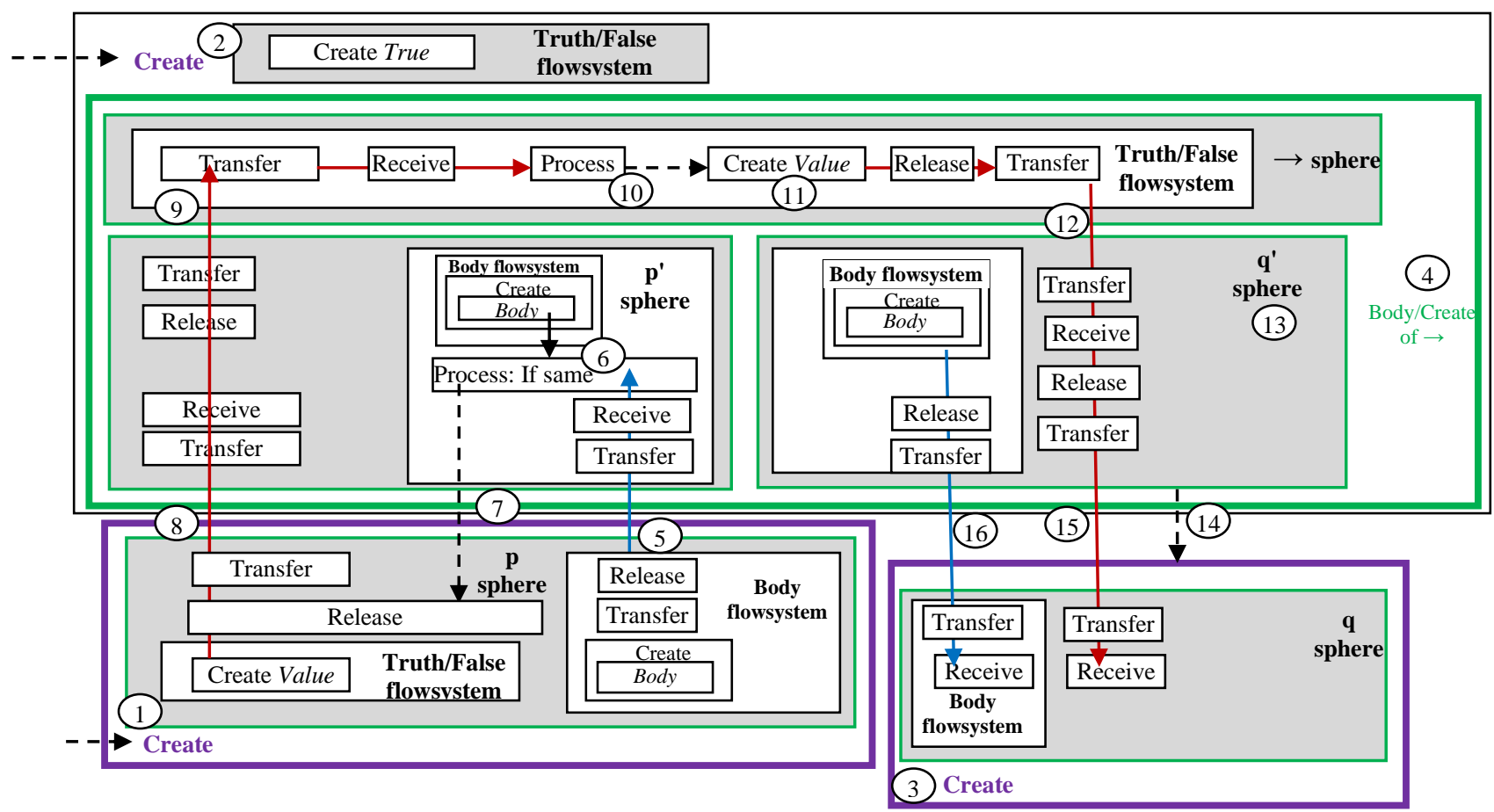

Fig. 6. FM representation of the MP: $\mathrm{p} \rightarrow \mathrm{q}, \mathrm{p}$ that produces the conclusion $\mathrm{q}$

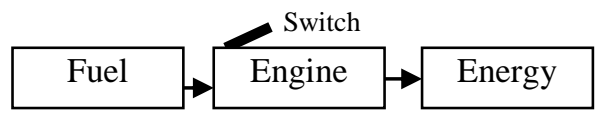

Fig. 7. Analogy of the activation of MP

The resultant truth-value is created (11) to flow (12) to the sphere of q' (13). This flow to the consequent causes it to trigger (14) the creation of q while "filling" it with a truthvalue (15) and body (16).

\section{LEWIS CARROLL}

This section shows an example using the FM representation to clarify the mechanism of the modus ponens by drawing it explicitly.

"What the Tortoise Said to Achilles" was written by Lewis Carroll in 1895 as a regress problem that arises from using MP as a deduction rule. It begins by considering the following logical argument:

A: "Things that are equal to the same are equal to each other"

B: "The two sides of this triangle are things that are equal to the same"

Therefore Z: "The two sides of this triangle are equal to each other"

Then, an objection is raised to deducing $\mathrm{Z}$ from $\mathrm{A}$ and $\mathrm{B}$, based on accepting that $\mathrm{A}$ and $\mathrm{B}$ are true, but not accepting the principle: if $\mathrm{A}$ and $\mathrm{B}$ are both true, then $\mathrm{Z}$ must be true. Accordingly, the premises are written as follows.

A: "Things that are equal to the same are equal to each other"
B: "The two sides of this triangle are things that are equal to the same"

$\mathrm{C}$ : "If $\mathrm{A}$ and $\mathrm{B}$ are true, $\mathrm{Z}$ must be true"

Therefore Z: "The two sides of this triangle are equal to each other"

However, it is possible to accept premise $\mathrm{C}$ while still refusing to accept the expanded argument. In this way, the list of premises continues to grow without end.

(1): "Things that are equal to the same are equal to each other"

(2): "The two sides of this triangle are things that are equal to the same"

(3): (1) and (2) $\Rightarrow(\mathrm{Z})$

(4): (1) and (2) and (3) $\Rightarrow(Z)$

(...

(n): (1) and (2) and (3) and (4) and ... and (n-1) $\Rightarrow(\mathrm{Z})$

Therefore (Z): "The two sides of this triangle are equal to each other."

Fig. 8 shows the FM representation of A, B, and Z. Now consider in the figure:

Refusing to deduce $\mathrm{Z}$ from $\mathrm{A}$ and $\mathrm{B}$ based on accepting that $\mathrm{A}$ and $\mathrm{B}$ are true, but not accepting the principle: if $\mathrm{A}$ and $\mathrm{B}$ are both true, then $\mathrm{Z}$ must be true.

The principle that, if $\mathrm{A}$ and $\mathrm{B}$ are both true, then $\mathrm{Z}$ must be true, is drawn explicitly in the figure as an application of Fig. 6 . It seems that refusal is related to the triggering that creates q (The two sides of this triangle are equal to each other). 


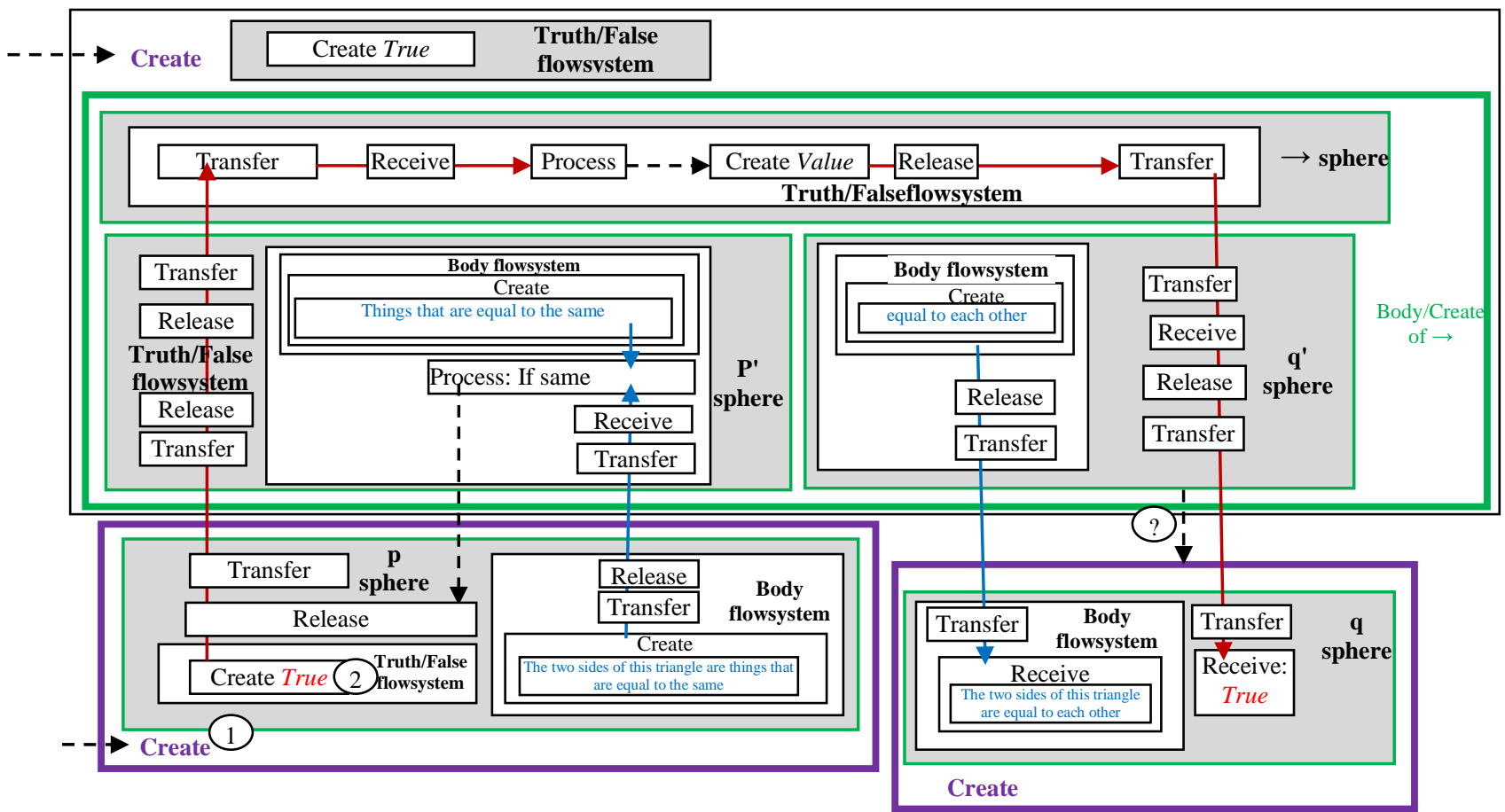

Fig. 8. FM representation of the MP: A: "Things that are equal to the same are equal to each other," B: "The two sides of this triangle are things that are equal to the same"; therefore Z: "The two sides of this triangle are equal to each other"

As explained when discussing $\mathrm{p} \rightarrow \mathrm{q}$ in Fig. 6, $\mathrm{p}$ is created (given), and its truth-value (2) flows to the implication $\rightarrow$ where the truth-value is created according to the truth table (3). Hence, q' is now "pregnant" with full q: It is true that the two sides of this triangle are equal to each other. Then, why disbelieve that q' "gives birth" to q? The whole process is a machine-like construction analogous to a machine designed to produce an output.

Another possible objection is disbelieving that the "machine" is designed correctly. What part, then, is the incorrect portion of the machine?

FM representation allows the mechanism of the modus ponens to be explicitly drawn, in contrast to being a "ghost" in such representations as the one shown in Fig. 9.

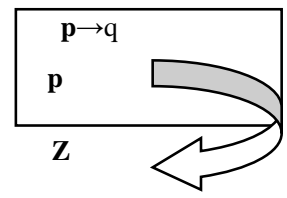

Fig. 9. Implicit representation of Modus ponens

\section{MCGEE's COUNTEREXAMPLE}

The FM representation can be used to diagram McGee's [5] counterexample mentioned in the introduction.

a) If a Republicans will win the election, then if Reagan will not win, Anderson will win.

b) A Republican will win the election. c) So, if Reagan will not win, Anderson will win. Or,
1. $\mathrm{p} \rightarrow(\mathrm{q} \rightarrow \mathrm{t})$
2. $\mathrm{p}$
3. $(\mathrm{q} \rightarrow \mathrm{t})$

Fig. 10 shows the corresponding FM representation. The first part, " $\mathrm{p} \rightarrow$ " appears as in the FM description shown in Fig. 6 ; however, starting with circle 12 , the truth-value result, this time, triggers (activates) another implication (blue box in the online version): $(\mathrm{q} \rightarrow \mathrm{t})$.

This, in turn, triggers the creation of q (circle 13 in Fig. 10), which is formulated from the body of $\mathrm{q}^{\prime}$ in the implication (14 and 15).

Accordingly, the true value of $\mathrm{q}^{\prime}$ (16) flows to the implication in $\left(\mathrm{q}^{\prime} \rightarrow \mathrm{t}^{\prime}\right)(17)$, where it is processed (18) to produce a truth-value according to the material implication truth table (19). Since $\left(\mathrm{q}^{\prime} \rightarrow \mathrm{t}^{\prime}\right)$ is true and $\mathrm{q}$ is true, then the generated truth-value is true. This truth-value flows to (the shell) $t^{\prime}$ (20) to trigger (21) the creation of $t$ using the body of $t^{\prime}$ (22).

Now, look at the "internal" MP:

$$
\mathrm{q} \rightarrow \mathrm{t}
$$

q

$\mathrm{t}$

The situation of $\left(\mathrm{q}^{\prime} \rightarrow \mathrm{t}^{\prime}\right)(12)$ being true does not necessarily originate from $\mathrm{q}$ is true, as shown in Table1. 


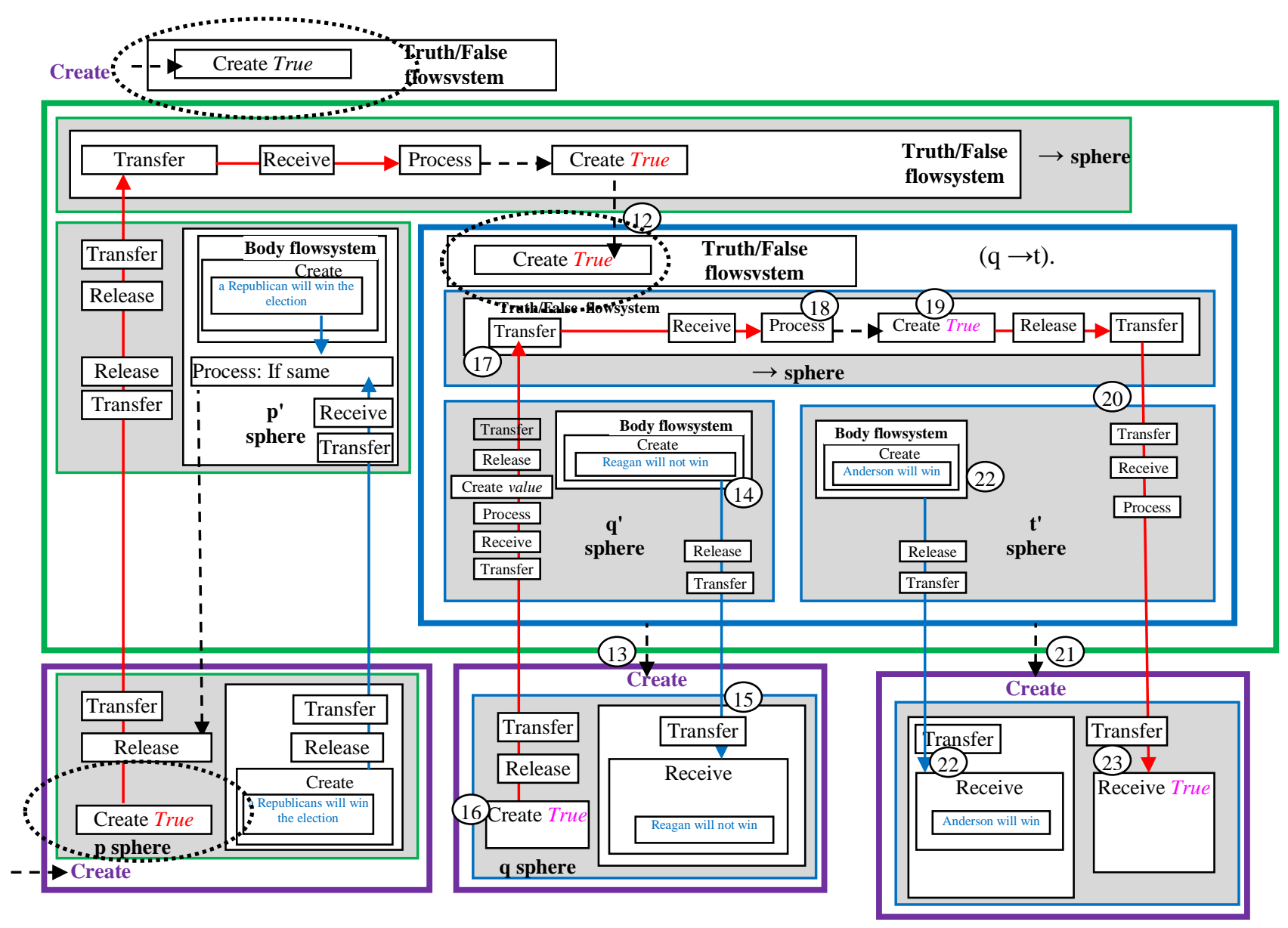

Fig. 10. FM representation of McGee's counterexample

TABLE I. TRUTH TABLE FOR “ $\mathrm{q} \rightarrow \mathrm{t}$ ” Is TRUE”

\begin{tabular}{|l|l|l|l|}
\hline & $\mathrm{q}$ & $\mathrm{t}$ & \multicolumn{1}{|c|}{$(\mathrm{q} \rightarrow \mathrm{t})$} \\
\hline $\mathbf{1}$ & true & true & true \\
\hline 2 & false & false & true \\
\hline 3 & false & true & true \\
\hline
\end{tabular}

The true value of $(\mathrm{q} \rightarrow \mathrm{t})$ "means" that $\mathrm{q}$ and $\mathrm{t}$ could be true or false, as shown in Table 1, relative to the assumed truthfulness of Reagan will not win. Accordingly, the implication $\mathrm{q} \rightarrow \mathrm{t}$ has three possibilities, all of which satisfy that $(\mathrm{q} \rightarrow \mathrm{t})$ is true:

(Row 1 in Table 1) A Republican will win the election $\rightarrow$ If Reagan will not win, Anderson will win

(Row 1 in Table 1) A Republican will win the election $\rightarrow$ If Reagan will win, Anderson will win

(Row 1 in Table 1) A Republican will win the election $\rightarrow$ If Reagan will win, Anderson will not win

Therefore, the MP should have been written as:

1) If a Republican will win the election, then

(If Reagan will not win, Anderson will win) V (If Reagan will win, Anderson will win) $V$ (If Reagan will win, Anderson will not win)

\section{2) A Republican will win the election}

3) (If Reagan will not win, Anderson will win) $V$

(If Reagan will win, Anderson will win) $V$ (If Reagan will win, Anderson will not win)

But for all $p_{1} \vee p_{2} \vee p_{3}, p_{1} \vee p_{2} \vee p_{3}$ is true if any of $p_{1}, p_{2}$, or $p_{3}$ is true. Accordingly, the consequent (3) is true because (If Reagan will win, Anderson will not win). In general, if $\mathrm{p}_{\mathrm{i}}$ is true then ( $\mathrm{p}_{\mathrm{i}} \mathrm{V}$ any false statement) is true. The controversy originated with the implication:

$\mathrm{p}$ is true $\rightarrow((\mathrm{q} \rightarrow \mathrm{t})$ is true $)$

Subsequently, we can substitute a false statement for $\mathrm{q}$ and $\mathrm{t}$ and still preserve the truthfulness. If fact, it is a valid deduction that:

1) If a Republican will win the election, then, if The moon is made of green cheese, Anderson will win

2) A Republican will win the election

3) (If The moon is made of green cheese, Anderson will win) [16].

This resulted from the definition of material implication 


\section{CONCLUSION}

This paper proposes a diagrammatic representation of modus ponens with the hope that such a representation can help to clarify issues related to rules of inference, specifically modus ponens. The advantage of this diagram-matic representation as a tool for understanding the reasoning process involved in this inference rule is demonstrated through examples. The results point to the viability of the approach. Further research may confirm such results.

\section{REFERENCES}

[1] B. Mates, Stoic Logic. Berkeley: University of California Press, 1953. ISBN 0-520-02368-4

[2] M. M. Dagli, "Modus ponens, modus tollens, and likeness," in Twentieth World Congress of Philosophy, August 10-15, Boston, USA, 1998.

[3] R. Reiter, "On reasoning by default," in Readings in Knowledge Representation, R. J. Brachman and H. J. Levesque, Eds. Los Altos, CA: Morgan Kaufman, 1985, pp. 402-410.

[4] H. Prakken, Logical Tools for Modelling Legal Arguments. Dordrecht: Kluwer, 1997.

[5] V. McGee, "A counterexample to modus ponens." J. Philos., vol. 82, pp. 462-471, 1985.

[6] W. Sinnott-Armstrong, J. Moor, and R. Fogelin, "A defense of modus ponens," J. Philos., vol. 83, pp. 296-300, 1986.
[7] E. J. Lowe, "Not a counterexample to modus ponens," Analysis, vol. 47, pp. 44-47, 1987.

[8] D. E. Over, "Assumptions and the supposed counterexamples to modus ponens," Analysis, vol. 47, pp. 142-146, 1987.

[9] C. Piller, "Vann McGee's counterexample to modus ponens," Philos. Stud., vol. 82, pp. 27-54, 1996.

[10] J. MacFarlane, "McGee on modus ponens," April 21, 2011. http://johnmacfarlane.net/142/mcgee.pdf

[11] S. Al-Fedaghi, "Schematizing proofs based on flow of truth values in logic," in IEEE International Conference on Systems, Man, and Cybernetics (IEEE SMC 2013), October 13-16, Manchester, UK, 2013.

[12] S. Al-Fedaghi, "Visualizing logical representation and reasoning," in 15th International Conference on Artificial Intelligence (ICAI'13), July 22-25, Las Vegas, USA, 2013.

[13] S. Al-Fedaghi, "Schematizing formulas for logic students," Int. J. Intell. Inform. Process., vol. 4, no. 4, pp. 27- 38, 2013.

[14] S. Al-Fedaghi, "On a flow-based paradigm in modeling and programming," Int. J. Advanced Comput. Sci. Appl. (IJACSA), vol. 6, no. 6, pp. 209-217, 2015.

[15] S. Al-Fedaghi, "Conceptualizing effects, and uses of information," in Information Seeking in Context Conference (ISIC 2008), September 1720, Vilnius. Lithuania, 2008.

[16] G. Sundholm, “'Inference versus consequence' revisited: inference, consequence, conditional, implication," Synthese, vol. 187, pp. 943956, 2012. 